\title{
Sonification system for aging Taiwanese people
}

\author{
Chih-Fang Huang ${ }^{1 *}$, and En-Ju Lin ${ }^{2}$ \\ ${ }^{1}$ Department of Information Communication, Yuan Ze University, Taoyuan, Taiwan \\ ${ }^{2}$ Department of Musicology, Heidelberg University, Heidelberg, Germany \\ *Corresponding author: Chih-Fang Huang (jeffh@saturn.yzu.edu.tw)
}

\begin{abstract}
Purpose Aging has become a significant problem in the recent years, due to the aging baby boomers. Most of the supervision systems use vision to perform care functions. However people usually cannot watch video all the time, therefore the proposed sonification for aging Taiwanese is used to express a particular situation at the children or nursing home, including both acoustic instrumental sound for the eventtriggered actions, and algorithmic composition to represent various environment situations. The nursing system can easily retrieve their seniors' situation anytime according to the transmitted audio signal and music via a network. Method The proposed Chinese music sonification system (CMSS) for older people can be implemented into two sections. Section 1: Eventtriggered sound is designed using Chinese instrumental audio samples performed by pipa, erhu, etc. Event-triggered sound is based on the input of various actions of aging people, such as watering, exercising, toileting, dining, sleeping, etc. Section 2: Computer-composed music, is designed using automated stochastic process of MIDI-data with various moods and situations mapped into music features, including musical scale, harmony, tonality, and rhythm. There are five Chinese music modes used for the musical scale. Among them Gong, Jiao, and Jiao are closer to the major mode in western music, while Shang and Yu are similar to the minor mode, therefore positive emotions will be mapped into a major-mode music, and the negative emotions will be correlated to a minor-mode music. Results \& Discussion CMSS is based on the hybrid event-triggered sound and computer composed music. Event-triggered sound is the pre-recorded discrete audio samples, while computer composed music is the calculated continuous MIDIrendered audio in real-time. A questionnaire is designed for the seniors to listen to the result of the CMSS, and most of them are satisfied with this innovated design. Action data is used for computer composed music calculation via CCD, RF ID, or any other wireless technology, and environment data is determined by sensors such as bio-feedback, temperature, etc., providing data for event-triggered sound. CMSS are implemented with computer music software Max/MSP, to perform CMSS for the seniors living in Taiwan.
\end{abstract}

Keywords: Chinese music sonification system, aging boom, algorithmic composition

\section{INTRODUCTION}

Aging problem is becoming significant in the recent years, about the baby boomers ${ }^{1}$. Most of the supervision systems use vision to perform elders' care function. However people usually cannot watch video all the time, therefore the proposed sonification ${ }^{2,3}$ for the aging Taiwanese is used to express situation for their children or nursing home in Taiwan, including both acoustic instrumental sound for the eventtriggered actions, and algorithmic composition ${ }^{4-7}$ to represent various environment situations. The nursing system can easily retrieve their elders' situation anytime according to the transmitted audio signal and music via network. Method is based on the proposed Chinese Music Sonification System (CMSS) for aging people including event-triggered sound recorded by Chinese instrumental audio samples, and computer composed music using automated stochastic process of MIDI data with various moods and situations mapped into music features. There are five Chinese music modes (Gong, Shang, Jiao, Zheng, and $\mathrm{Yu})^{8}$ used for the musical scale. Among them Gong, Jiao, and Jiao are closer to major mode in western music, while Shang and Yu are similar to minor mode, therefore the positive emotion will be mapped into a major-mode music, and the minor emotion will be correlated to a minor-mode music.

The proposed computer composed music using automated background music composition technique, which integrates the new trends of algorithmic composition, music theory, human-computer-interface $(\mathrm{HCl})$, emotion, tension, etc. Hopefully the conventional background music composition can be not only played as a constant loop, but also performed via the scene settings of the content related to aging people. The research is based on algorithmic composition to generate the various music parameter distributions, therefore the generated music can be composed automatically according to the variation of the scenarios. The discussion of various scenarios mapped into a 2-D emotion coordinate $9^{9,10,11}$ to generated affective-related music is presented too.

CMSS is based on the hybrid event-triggered sound and computer composed music. Event-triggered sound is the pre-recorded discrete audio samples, while computer composed music is the calculated continuous MIDI-rendered audio in real-time. Action 
data is used for computer composed music calculation via user's selection currently, or in the future via CCD, Microsoft Kinect ${ }^{12}$, or any other wearless technologies, and environment data is determined by sensors such as bio-feedback, temperature, etc., providing data for event-triggered sound. CMSS is implemented with computer music software, to perform CMSS for the elders living in Taiwan.

\section{METHOD}

Since melody and styled accompaniment can be generated by the proposed automated composition for the computer composed music, a 2-D emotion map, as shown in Fig. 1, is used to compose music automatically with the emotion-music feature mapping and the proposed emotion trajectory which can be related to the situations of aging people.

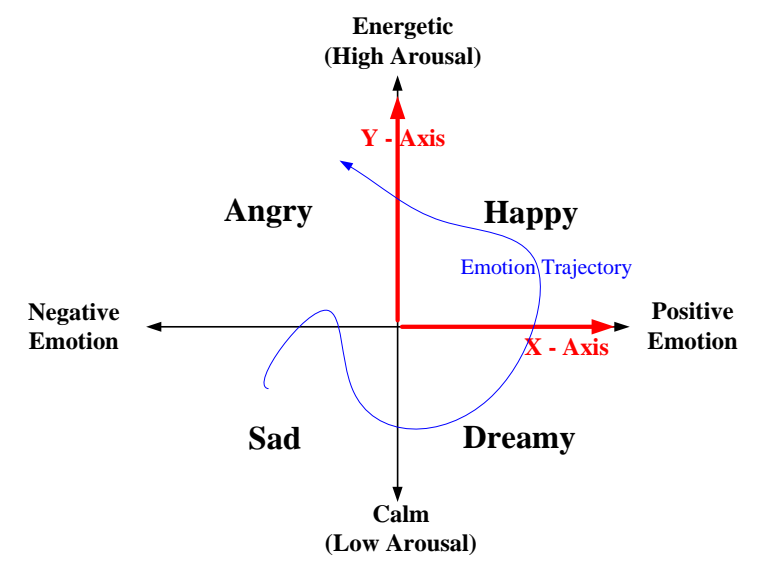

Fig.1. 2-D Emotion Map for CMSS System

The feature selection is also important in music style classification and there is a catalogue of features that can be used to classify was defined ${ }^{13}$. In the catalogue of features, we used two categories, timbre and texture, in our rules. Timbre and texture will be the essential factor of our arranged accompaniment. Furthermore, rhythm is always discussed when it talks to music style. Three types of features will be introduced in this paper. Firstly, pitch range is the only one feature based on pitch that we choose to use in our accompaniment generation. It means the difference in semitones between the highest and the lowest note. In general, most features based on pitch in classification are defined to be used in the melody. However, we concern about the features of accompaniment in this part. So we use pitch range only to place restrictions on pitch of our accompaniment. Secondly, many types of music are recognized by their own rhythm pattern. When people hear the particular rhythm pattern, they will recall that kind of music on the moment. We use rhythm complexity which is defined as the measures closest to human performance difficulty in rhythm ${ }^{14}$. Thirdly, timbre or instrument selection plays an important role in accompaniment. Instrument timbre will be affect ac- companiment arrangement profoundly. All features based on timbre are described as followed: (1) Orchestration: A features array with one entry for each of the $128 \mathrm{GM}$ instruments presents which of the 128 MIDI instruments are played. (2) Number of Pitched Instruments: Total number of General MIDI instruments that were used to play at least one note. (3) Number of Un-pitched Instruments: Total number of MIDI Percussion Key Map instruments that were used to play at least one note.

In music, texture determines the overall quality of a piece. Two features based on texture are described as followed: (1) Maximum Number of Independent Voices: Maximum number of different channels in which notes have sounded simultaneously. (2) Simultaneity: Maximum number of notes sounding simultaneously.

Method As shown in Fig. 2, the proposed CMSS for elder people can be implemented into two parts. Part 1: Event-triggered sound, is designed using Chinese instrumental audio samples performed by pipa, erhu, etc. Event-triggered sound is based on the input of various actions of aging people, such as watering, exercising, toileting, dining, sleeping, etc. Part 2: Computer composed music, is designed using automated stochastic process of MIDI data with various moods and situations mapped into music features, including musical scale, harmony, tonality, and rhythm.

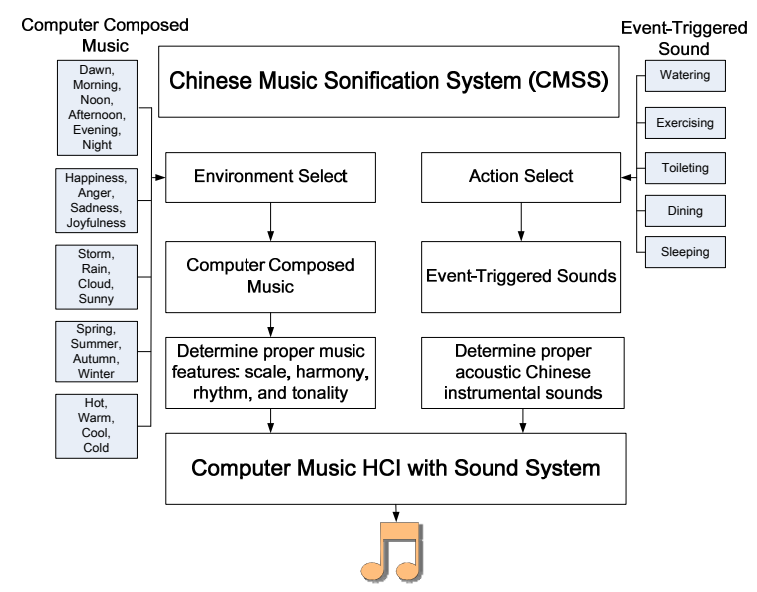

Fig.2. CMSS System for the elder Taiwanese People

\section{Implementation}

The implementation of event-triggered sound is basically a set of pre-recorded sound performed and prerecorded by Chinese instruments, while computer composed music is designed by computer program. The automated composition techniques is applied for the computer generated music using a 2-D emotion coordinate system to map the valence and arousal data from the state of the emotion trajectory into music features, including rhythmic roughness, temp, articulation, mode brightness, harmony complexity, and pitch register, as shown in Table 1. 
Table 1. The Mapping Relation between Music Features and Emotion Valence / Arousal

\begin{tabular}{|c|c|}
\hline Valence & Low $\leftarrow$ Valence $\rightarrow$ High \\
\hline \multirow{2}{*}{$\begin{array}{c}\text { Music } \\
\text { features }\end{array}$} & Smooth $\leftarrow$ rhythmic roughness $\rightarrow$ rough \\
\cline { 2 - 2 } & Slow $\leftarrow$ tempo $\rightarrow$ fast \\
\cline { 2 - 2 } Arousal & Lowato $\leftarrow$ articulation $\rightarrow$ staccato \\
\hline \multirow{2}{*}{$\begin{array}{c}\text { Music } \\
\text { features }\end{array}$} & Low $\leftarrow$ mode brightness $\rightarrow$ High \\
\cline { 2 - 2 } & Low $\leftarrow$ harmony complexity $\rightarrow$ High \\
\cline { 2 - 2 } & low $\leftarrow$ pitch register $\rightarrow$ high \\
\hline
\end{tabular}

In this paper Chinese mode is used for music generation, and "mode" refers to the variance between major and minor which is well defined in the literatures of the western music theory. Modulation in parallel keys between major and minor can be used to make the generated music performed from positive to negative emotion, where Chinese Gong mode is selected as the "major key", and Chinese Yu mode is used as the "minor key" ${ }^{\text {"11 }}$. An example of the parallel keys is specified in Fig. 4 which specifies two scales in the scores to implement for our proposed CMSS.

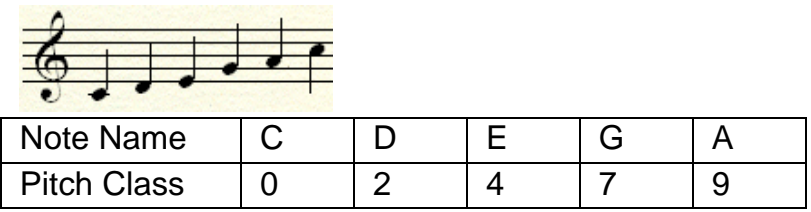

Fig.3. C-Gong Mode Scale Definition

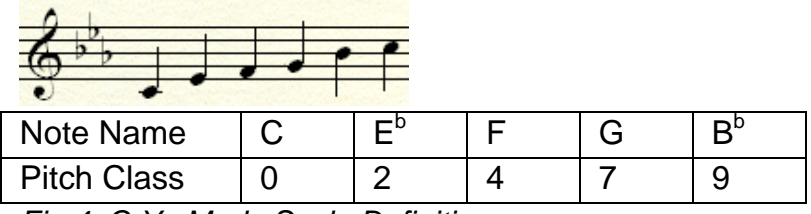

Fig.4. C-Yu Mode Scale Definition

The only difference between the major scale and the harmonic scale is the $3^{\text {rd }}$ note and the $6^{\text {th }}$ note, which can be manipulated with the probability control to guide the "trending" for major and minor keys. With using this point, when the data value of $X$ coordinate is big enough, then the major key can be generated. When the $X$ value is decreased, then the appearance rate of E-nature, $3^{\text {rd }}$ note of the major key, and A-nature, $6^{\text {th }}$ note of the major key, is lowered, and the E-flat, $3^{\text {rd }}$ note of the minor key, and B-flat, $7^{\text {th }}$ note of the minor key, begins to show up after the $X$ value is across the $X$ axis to the negative.

In addition to music scale, more music features can be used to perform the probability control for automated composition, including chord progression, rhythm, etc.. In Fig. 5 Markov Chain is used to control the first-order states transition (S1, S2,.., Sn), and the correspondent probabilities P1, P2, .., Pn can be assigned according to the emotion-music features mapping relation, as shown in Fig. 5.

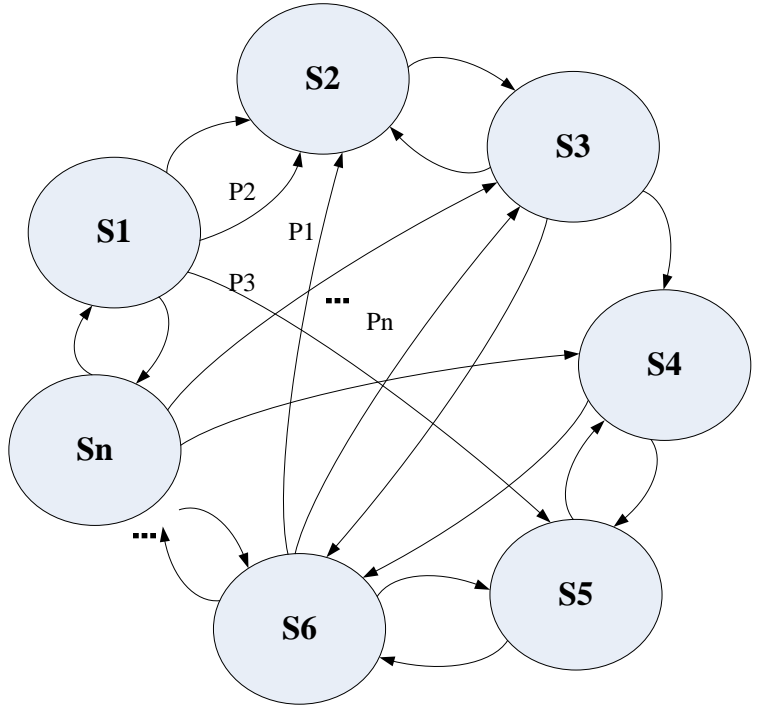

Fig.5. Markov System for the CMSS Automated Music Composition

Finally the emotion-driven music data can be mapped into the CMSS environmental situated data according to the scenario of the aging people, including weather, temperature, season, and time, as shown in Table 2, and then the CMSS automated music composition can be implemented.

Table 2. CMSS Environment - Emotion Mapping

\begin{tabular}{|l|l|l|l|l|}
\hline Env. & Happy & Angry & Sad & Dreamy \\
\hline Weather & Sunny & $\begin{array}{l}\text { Heavy } \\
\text { Rain }\end{array}$ & $\begin{array}{l}\text { Small Rain / } \\
\text { Cloudy }\end{array}$ & Normal \\
\hline Temperature & Hot & Hot & Cold & Warm \\
\hline Season & Summer & Summer & $\begin{array}{l}\text { Autumn / } \\
\text { Winter }\end{array}$ & Spring \\
\hline Time & Morning & Noon & Dusk & Night \\
\hline
\end{tabular}

\section{Result}

The result of the CMSS is currently simulated and obtained by the mapping from action and environment select from aging people, and the scenarioemotion data mapping. Currently most of the aging people subjects satisfy the result of CMSS. In the future more sensor-based data including CCD or Microsoft Kinect can be used to implement the context-aware system for CMSS. 


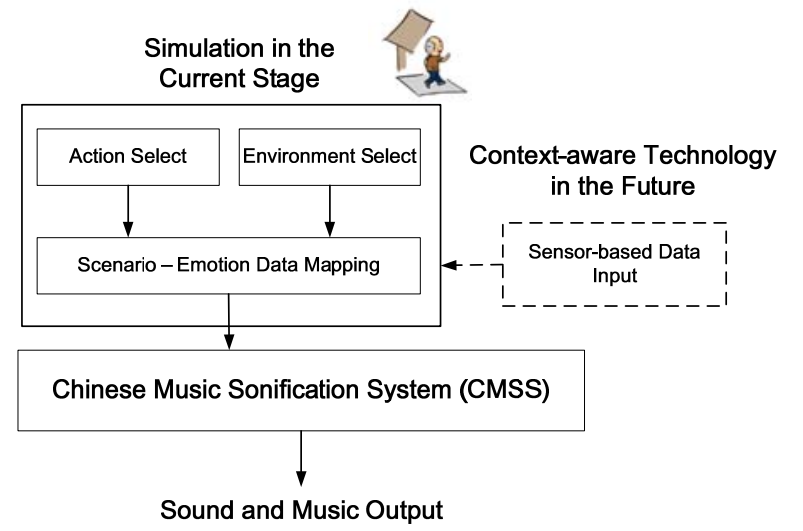

Fig.6. The Result of CMSS Output including Both Current Simulation and the Future Context-aware Technology

\section{ConcLusion}

The proposed CMSS shows the potential to perform the sonification function of aging people situation for either care centers of children without the need of supervision by eyes. In the future the sensor-based technology will be integrated with CMSS based on our simulation result, to perform more efficient and accurate function for our aging people.

\section{ACKNOWLEDGMENT}

The authors would like to appreciate the support from National Science Council projects of Taiwan: NSC 100-2627-E-155 -001 and NSC99-2410-H-155 $-035-\mathrm{MY} 2$.

\section{References}

1. Drummonds, H., "The Aging of the Boomers and the Coming Crisis in America's Changing Retirement and Elder Care Systems", Lewis \& Clark Law Review, Vol. 11(2), pp. 267-303, 2007.

2. Ballas, A., "Delivery of Information through Sound", Auditory Display: Sonification, Audification and Auditory Interfaces., Ed. Gregory Kramer, Reading, MA: Addison-Wesley, pp 79-94, 1994.

3. Ballas, A., "Delivery of Information Through Sound in Auditory Display", Sonification, Audification and Auditory Interfaces, Ed. Gregory Kramer, Reading, MA: Addison-Wesley, pp 79-94, 1994.

4. Winsor, P., "Automated Music Composition", Denton, TX: University of North Texas Press, 1992.

5. Supper, M., "A Few Remarks on Algorithmic Composition", Computer Music Journal, Vol. 25(1), pp. 48-53, 2001.

6. Barlow, C., "Autobusk: An Algorithmic Real-time Pitch and Rhythm Improvisation Programme.", Proceedings of the International Computer Music Conference, San Francisco: International Computer Music Association, pp. 166-168, 1990.

7. Cope, D., "Virtual Music: Computer Synthesis of Musical Style", The MIT Press, 2004.
8. Ho, L.T., Han, K.H., "On Chinese Scales and National Modes", Asian Music, Vol. 14(1), pp.132-154, 1982.

9. Wagner, J., Kim, J., André, E., "From Physiological Signals to Emotions: Implementing and Comparing Selected Methods for Feature Extraction and Classification", IEEE International Conference on Multimedia \& Expo, 2005.

10. Russell, J.A., "A Circumplex Model of Affect", Journal of Personality and Social Psychology, Vol. 39 (6), pp. 1161-1178, 1980.

11. Livingstone, S.R., Brown, A.R., "Dynamic Response: Real-Time Adaptation for Music Emotion". Proceedings of the Second Australasian Conference on Interactive Entertainment, 2005.

12. Gallo, L., "Controller-free Exploration of Medical Image Data: Experiencing the Kinect", ComputerBased Medical Systems (CBMS), 24th International Symposium on, pp. 27-30, June 2011.

13. McKay, C., "Automatic genre classification of MIDI recordings," M.A. Thesis, McGill University, Canada, 2004.

14. Thul, E., Godfried T.T., "On the Relation Between Rhythm Complexity Measures and Human Rhythmic Performance", Proceedings of the Canadian Conference on Computer Science and Software Engineering, Editor: Bipin C. Desai, Concordia University, Montreal, Canada, May 12-13, pp. 199-204, 2008. 